\title{
Integrating Semantic Web and Language Technologies to Improve the Online Public Administrations Services
}

\author{
Marta Gatius Meritxell González \\ TALP Research Center (UPC) \\ Jordi Girona, 1-3, Campus Nord UPC \\ 08034 Barcelona, Spain \\ 34934137797 \\ \{gatius,mgonzalez\}@|si.upc.edu
}

\author{
Sheyla Militello \\ Loquendo S.p.A \\ Via Nole, 55 \\ 10149 Torino, Italy \\ 390117576413
}

\author{
Pablo Hernández \\ SUN Microsystems \\ Avda. Diagonal, 640 \\ 0817 Barcelona, Spain \\ 34934804000
}

\begin{abstract}
In this paper, we describe how domain ontologies are used in a dialogue system guiding the user to access web public administration contents. The current implementation of the system supports speech (through the telephone) and text mode in different languages (English, Spanish, Catalan and Italian).
\end{abstract}

\section{Categories and Subject Descriptors}

H.5.2. [Information Interfaces]: User Interfaces - Natural language, Voice I/O, User interface management systems (UIMS).

General Terms: Management, Design, Human Factors.

Keywords: Dialogue systems, ontologies, web usability, egovernment.

\section{INTRODUCTION}

Most of public administrations (as well as most of businesses and other entities) offer customers web self-service, being the benefits of self-service extremely tangible and measurable. However, many consumers prefer to use the telephone. Call Centers are a good solution, but the labour costs of human agents are expensive. A better solution seems to be integrating interaction self-service channels (web and telephone) and human agents.

Dialogue Systems (henceforth DSs) seems a good approach to self-service because they could render more efficient current services with important performance shortcomings. Additionally, the voice mode improves web usability, especially for people with different types of impairments (visual, physical or cognitive). Other advantages of the language modes are the following:

1. They are friendly and easy to use.

2. They support different types of interactions (menus, form filling and commands).

3. They can tackle complex questions whose answer will require processing information from different sources.

4. They can be integrated with other modes.

5. They are adaptable to different types of users (different languages, skills, ages and cultural sensitivity).

Speech recognition in open domain has still performance problems. For this reason, the speech mode is basically used in restricted domain systems. However, application-restricted DSs

Copyright is held by the author/owner(s).

$W W W$ 2006, May 23-26, 2006, Edinburgh, Scotland.

ACM 1-59593-323-9/06/0005. are expensive to develop and difficult to adapt to new domains. The definition of the standard language VoiceXML (an XMLbased mark-up language explicitly dedicated to the development of internet-powered telephone applications) reduces the cost of developing DSs and facilitates technology integration. Nevertheless, dialogues, grammars and system messages still have to be built for each service.

In this paper we describe how application-specific ontologies are used to improve both the engineering and functionalities features in the DS developed in the European project HOPS ("http://www.hops-fp6.org"). This system was designed to enhance the usability and accessibility of online public services and information. The current implementation of the system supports speech (through the telephone) and text mode in several languages (English, Spanish, Catalan and Italian).

\section{ARCHITECTURE OF THE SYSTEM}

The architecture of the system is shown in Figure 1. The system is composed of three layers.

1. Presentation. It controls interaction with the user.

a. The Audio Web Server connected with a VoiceXML Platform acting as Voice Browser.

b. The Text Server and the Natural Language Analyzer for text interaction.

2. Dialogue Management. It controls the dialogue flow.

a. The Application Manager, controlling the language and the service identification.

b. The Dialogue Manager, controlling dialogue.

3. Knowledge. It manages data for the application.

a. The Ontology Manager, for ontology access.

b. Action \& Query Manager, for backend access.

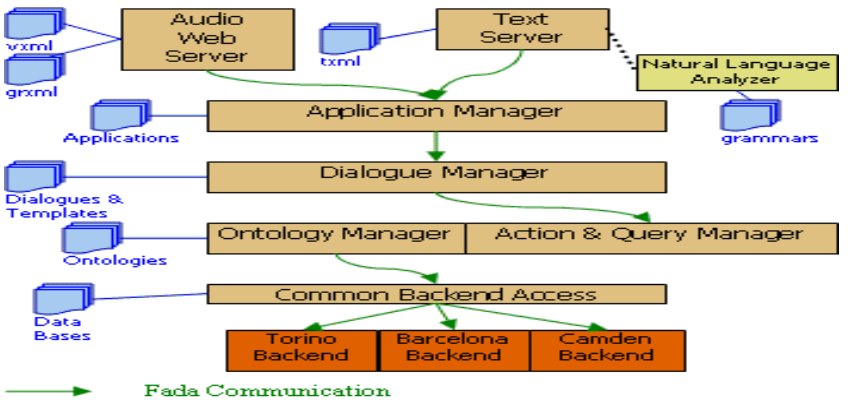

Figure 1. The architecture of the system

The different components of the system are interrelated between themselves composing a service oriented architecture. 
The ontologies are represented in the standard language OWL. This choice is justified by several reasons:

1. It is a standard language recommended by the $\mathrm{W} 3 \mathrm{C}$.

2. It is more expressive than other standard languages.

3. Its reasoning capabilities are enough for the system.

4. It has tool support (including reasoners).

\section{THE USE OF ONTOLOGIES}

The number of DSs using ontologies modeling the service knowledge involved in communication $([1,2,3,4])$ is increasing. In several situations ontologies are specially appropriate:

1. In complex and changing domains.

2. In mutli-modal and/or multilingual systems (ontologies provide a common semantics).

3. In multi-domain systems (ontologies facilitate the reuse of knowledge common to different services).

Current research in semantic web for e-government is focused in defining a common infrastructure for semantic interoperability, using more or less formalized domain ontologies. This framework could be used to address the mass-scale deployment of new online public services accessible by the citizens. Our work is focused on DSs using ontologies to guide the user when accessing public administration web services because there is no much research in this area and we think it could be useful. In this context, we are planning to link the ontologies modeling the services supported by our system to the Electronic Service Delivery, a number of related structured category lists related to web services that are used as standards by local governments in the United Kingdom.

In the DS we have developed, dialogues, grammars and system messages for speech and textual interaction they are obtained from the ontology modeling the service tasks. In this ontology, the service tasks are represented by a set of concepts described by attributes. These attributes represent the information required/resulting when performing the service tasks. As this information basically consists of the input and output task parameters, the attributes describing the ontology concepts can be obtained semi-automatically from the service back-end.

Dialogues consist mainly in asking/giving the values of the conceptual attributes. These attributes can also be linked to domain ontologies providing domain-specific semantics. Preconditions are associated with the ontology concepts to drive the information that has to be asked to the user at each state of the dialogue. Using this ontology the dialogue manager can support different types of dialogues: system-driven (taking the initiative according to the attributes representing the information needed from the user) and mixed-initiative dialogues (verifying the relationship among the attributes values given by user).

\subsection{Sample Services}

We have selected two main areas as sample service areas for piloting, these being the Cultural Agenda and the Large Objects Collection Service. The Cultural Agenda is an informational service giving information about the cultural events in a city. The attributes describing the event search are those parameters of the query back-end involved in the communication: one/list (search for a specific event or a list), title, type, subtype, performers, location, address, starting and ending dates and description.

The Large Objects Collection is a transactional service for collecting large objects. A fragment of the ontology representing this service can be seen in Figure 2. The three tasks performed by this service are represented in three separated concepts:
Information, Collection and Cancellation. The collection task sets a date for collection. The attributes describing this concept correspond to the service back-end parameters (object type, user type, address, name, telephone, date). The attribute objectype (representing the type of the object to be collected) is linked to specific taxonomies providing all its possible values. Figure 2 shows how one of these taxonomies (a furniture taxonomy) is used when creating the grammar modeling the user's intervention describing the object that has to be collected.

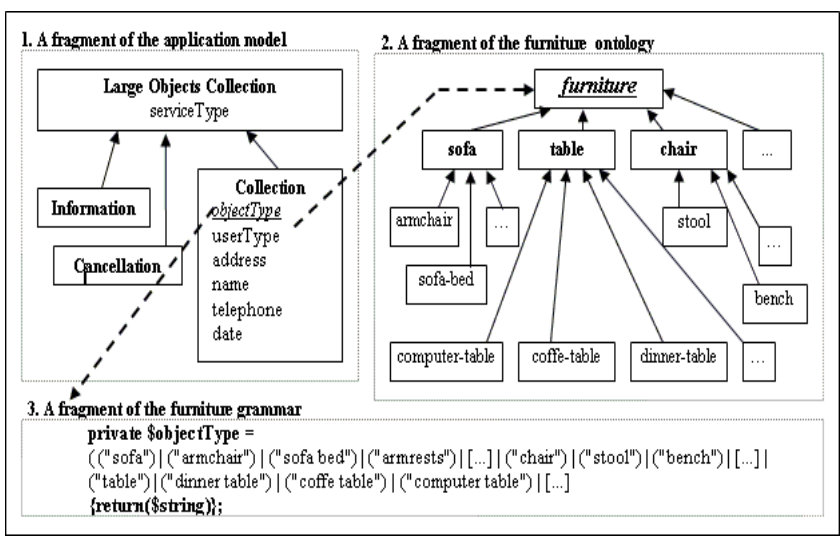

Figure 2. The Large Objects Collection

\section{CONCLUSIONS AND FUTURE WORK}

We have presented the DS developed to deal with the delivery of information and the transaction of public services in the local administration. We have described how ontologies representing the application-specific knowledge are used in this system to improve both the dialogue management as well as the generation of application-restricted dialogues, grammars and system's messages for speech and text mode in several languages.

We are planning to test the system with real users. Future work in the project will include integrating the ontologies modeling the service in a general e-government infrastructure including semantic web technologies. Future improvements on the system capabilities will be achieved by incorporating semantic web techniques, such as service discovering and composition.

\section{ACKNOWLEDGMENTS}

This work has been supported by the EU IST FP6 project HOPS (IST-2002-507967).

\section{REFERENCES}

[1] Dupriez, C. Multilingual Vocal Access to Databases: the Central Role of Ontology Management in VocaBase. Sixth International Protégé Workshop, England, 2003.

[2] Milward, D., Beveridge, M. Ontology-Based Dialogue Systems. Third Workshop on Knowledge and Reasoning in Practical Dialogue Systems, 2003.

[3] Wahlster, W. SmartKom: Symmetric Multimodality in an Adaptive and Reusable Dialogue Shell. Human Computer Interaction Status Conference, 2003.

[4] Wilcock, G., Jokinen, K. Generating Responses and Explanations from RDF/XML and DAML+OIL. Third Workshop on Knowledge and Reasoning in Practical Dialogue Systems, 2003. 